\title{
Dimensionality Reduction for Point Feature SLAM Problems with Spherical Covariance Matrices
}

\author{
Heng Wang ${ }^{\mathrm{a}}$, Shoudong Huang ${ }^{\mathrm{b}}$, Kasra Khosoussi ${ }^{\mathrm{b}}$, Udo Frese ${ }^{\mathrm{c}}$, \\ Gamini Dissanayake ${ }^{\mathrm{b}}$, Bingbing Liu ${ }^{\mathrm{a}}$ \\ ${ }^{a}$ Institute for Infocomm Research, Agency for Science, Technology and Research (A*STAR), Singapore. \\ ${ }^{\mathrm{b}}$ Centre for Autonomous Systems, Faculty of Engineering and Information Technology, University of Technology, Sydney, Australia. \\ ${ }^{\mathrm{c}}$ German Research Center for Artificial Intelligence (DFKI) in Bremen, Germany.
}

\begin{abstract}
The main contribution of this paper is the dimensionality reduction for multiple-step 2D point feature based Simultaneous Localization and Mapping (SLAM), which is an extension of our previous work on one-step SLAM (Wang, Huang, Frese \& Dissanayake 2013). It has been proved that SLAM with multiple robot poses and a number of point feature positions as variables is equivalent to an optimization problem with only the robot orientations as variables, when the associated uncertainties can be described using spherical covariance matrices. This reduces the dimension of original problem from $3 m+2 n$ to $m$ only (where $m$ is the number of poses and $n$ is the number of features). The optimization problem after dimensionality reduction can be solved numerically using the unconstrained optimization algorithms. While dimensionality reduction may not provide computational saving for all nonlinear optimization problems, for some SLAM problems we can achieve benefits such as improvement on time consumption and convergence. For the special case of two-step SLAM when the orientation information from odometry is not incorporated, an algorithm that can guarantee to obtain the globally optimal solution (in the maximum likelihood sense) is derived. Simulation and experimental datasets are used to verify the equivalence between the reduced nonlinear optimization problem and the original full optimization problem, as well as the proposed new algorithm for obtaining the globally optimal solution for two-step SLAM.
\end{abstract}

Key words: Multiple-step SLAM; least squares; dimensionality reduction.

\section{Introduction}

For more than 15 years, Simultaneous Localization and Mapping (SLAM) has been a key problem in robotics (Bailey \& Durrant-Whyte 2006). As a result, many algorithms have been proposed to solve SLAM in various forms. In the state-of-the-art approaches, under the assumption of (independent) Gaussian noise, the SLAM problem is formulated as a sparse non-linear least squares (NLLS) problem over $m$ robot poses and $n$ features' positions $(3 m+2 n$ variables in 2D) (Dellaert \& Kaess 2006). Newton-based iterative solvers such as Gauss-Newton and

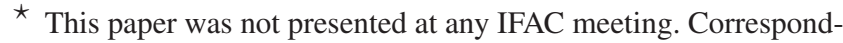
ing author H. Wang. Tel. +65-64082790. Fax +65-67761378.

Email addresses: wang-h@i2r.a-star.edu.sg (Heng

Wang), Shoudong.Huang@uts.edu.au (Shoudong

Huang), Kasra.Khosoussi@uts.edu.au (Kasra

Khosoussi), udo.frese@dfki. de (Udo Frese),

Gamini.Dissanayakeduts.edu.au (Gamini

Dissanayake), bliu@i2r.a-star.edu.sg (Bingbing Liu).
}

Levenberg-Marquardt are among the most popular algorithms for solving this NLLS. The sparseness of this NLLS is a consequence of (i) conditional independence of features given the robot poses, (ii) limited range of sensors, and finally (iii) uncorrelated measurement noise. Exploiting this inherent property of SLAM problems is a key characteristic of many of the modern solvers (Kaess, Johannsson, Roberts, Ila, Leonard \& Dellaert 2012)(Kummerle, Grisetti, Strasdat, Konolige \& Burgard 2011)(Huang, Wang \& Dissanayake 2008).

It is now well-known that the SLAM problem becomes considerably easier to analyse when the noise covariance matrices are spherical (Wang et al. 2013). In Huang, Lai, Frese \& Dissanayake (2010), the authors reported an unexpected convergence of vanilla Gauss-Newton algorithm to the optimal solution from random initial guesses in high-dimensional SLAM problems when the noise covariance matrices are spherical. Similarly, in the tree-based network optimizer (TORO) (Grisetti, Stachniss, Grzonka \& Burgard 2007), good convergence results from bad initial values are reported 
for large pose-graphs when the noise covariance matrices are spherical. More recently, Carlone (Carlone 2013) addressed the convergence of Gauss-Newton algorithm in pose-graph SLAM. Under the assumption of spherical noise covariance matrices, a conservative estimate for the basin of attraction of the ML estimate in pose-graph SLAM was derived. In Wang et al. (2013), Huang, Wang, Frese \& Dissanayake (2012), Wang, Hu, Huang \& Dissanayake (2012), authors studied the ML objective function in "one-step SLAM" when the noise covariance matrices are spherical. They show that minimizing the ML objective function (in one-step SLAM) can be reduced to a one-dimensional optimization problem over the robot orientation. Furthermore, it is shown that the reduced problem has a unique minimizer unless the noise is extremely large.

In this paper we extend the results of Wang et al. (2013), Huang et al. (2012), Wang et al. (2012) along two directions: (i) we show that the ML estimate in general ( $m$-step) featurebased SLAM problems can be obtained by solving a NLLS problem over only $m$ variables (i.e., the robot orientations in $m$ poses), in particular, the structure of the problem is closely related to the incidence matrix of the directed graph of the SLAM problem, and (ii) based on this result, we develop an algorithm that can be guaranteed to find the globally optimal solution to the 2-step SLAM when the orientation information in odometry is not used.

This paper is organized as follows. In Section 2, the ML estimate in SLAM is formulated as the solution to a NLLS problem. Section 3 introduces the definition of spherical matrices and derives an alternative SLAM formulation when covariance matrices are spherical. In Section 4, it is shown that the $m$-step ( $m \geq 2$ ) SLAM problem is equivalent to another NLLS over only the robot orientations. In Section 5 , we show that the globally optimal solution to a special case of two-step SLAM problem can be obtained by finding the roots of a polynomial with degree 6 . In Section 6, examples are presented to illustrate the benefits of dimensionality reduction. Finally Section 7 concludes the paper.

Notations: Throughout the paper, $\otimes$ denotes the Kronecker product, superscript $T$ and -1 stand for, respectively, the transposition and the inverse of a matrix; $C \succ D$ means that matrix $C-D$ is positive definite; $I$ and $I_{n}$ denote the identity matrix with compatible dimension and dimension $n, 0$ represents the zero matrix with compatible dimension, and $\|e\|_{C}^{2}=e^{T} C e$, where $C \succ 0$ and $e$ is a vector. $\operatorname{wrap}(\cdot)$ is the function that maps an angle to its equivalent angle in $(-\pi, \pi]$. The symbol $\operatorname{diag}\left(C_{1}, \ldots, C_{n}\right)$ denotes a blockdiagonal matrix whose diagonal blocks are $C_{1}, \ldots, C_{n}$.

\section{Problem Formulation}

Suppose $n$ 2D point features $\left\{f_{i}\right\}_{i=1}^{n}$ are observed from a sequence of $m+12 \mathrm{D}$ robot poses $\left\{r_{i}\right\}_{i=0}^{m}$. We use $Z_{k}^{i}$ to denote the observation made from pose $r_{i}$ to feature $f_{k}$. We use $O_{i}$ $(1 \leq i \leq m)$ to denote the odometry measurement between pose $r_{i-1}$ and pose $r_{i}$. Both the odometry and observations are corrupted by zero-mean Gaussian noises with covariance matrices $P_{Z_{k}^{i}}$ and $P_{O_{i}}$, respectively. $X_{f_{k}}=\left(x_{f_{k}}, y_{f_{k}}\right)^{T}$ denotes the position of feature $f_{k} . X_{r_{i}}=\left(x_{r_{i}}, y_{r_{i}}\right)^{T}$ denotes the position of robot pose $r_{i}$ while $\phi_{r_{i}}$ denotes the orientation of robot pose $r_{i}$. The coordinate frame is defined by the robot pose $r_{0}$. That is, $X_{r_{0}}=(0,0)^{T}$ and $\phi_{r_{0}}=0 . R\left(\phi_{r_{i}}\right)$ is the rotation matrix corresponding to $\phi_{r_{i}}$ defined by:

$$
R\left(\phi_{r_{i}}\right) \triangleq\left[\begin{array}{cc}
\cos \phi_{r_{i}} & -\sin \phi_{r_{i}} \\
\sin \phi_{r_{i}} & \cos \phi_{r_{i}}
\end{array}\right]
$$

The non-linear least squares (NLLS) SLAM formulation (Dellaert \& Kaess 2006) uses the odometry and observation information to estimate the state vector containing all the robot poses and all the feature positions

$$
X \triangleq\left(X_{f_{1}}^{T}, \cdots, X_{f_{n}}^{T}, X_{r_{1}}^{T}, \phi_{r_{1}}, \cdots, X_{r_{m}}^{T}, \phi_{r_{m}}\right)^{T}
$$

and minimizes the negative log-likelihood function

$$
\begin{aligned}
F(X)= & \sum_{i=0}^{m} \sum_{j=1}^{n_{i}}\left\|Z_{k_{i j}}^{i}-H^{Z_{k_{i j}}^{i}}(X)\right\|_{P_{z_{i j}^{i}}^{-1}}^{2} \\
& +\sum_{i=1}^{m}\left\|O_{i}-H^{O_{i}}(X)\right\|_{P_{O_{i}}^{-1}}^{2}
\end{aligned}
$$

where $O_{i}(1 \leq i \leq m)$ are odometries, $Z_{k_{i j}}^{i}$ are observations (assume $n_{i}$ features are observed from robot pose $r_{i}$ and $k_{i j}$ is the global index of the $j$-th feature observed from pose $\left.r_{i}\right)$, and $P_{O_{i}}$ and $P_{Z_{k_{i j}}^{i}}$ are the corresponding covariance matrices.

In the above least squares SLAM formulation, $H^{Z_{k}^{i}}(X)$ and $H^{O_{i}}(X)$ are the corresponding functions relating $Z_{k}^{i}$ and $O_{i}$ to the state $X$. An odometry measurement is a function of two poses $\left(X_{r_{i-1}}^{T}, \phi_{r_{i-1}}\right)^{T}$ and $\left(X_{r_{i}}^{T}, \phi_{r_{i}}\right)^{T}$ and is given by

$$
H^{O_{i}}(X)=\left[\begin{array}{c}
R\left(\phi_{r_{i-1}}\right)^{T}\left(X_{r_{i}}-X_{r_{i-1}}\right) \\
\operatorname{wrap}\left(\phi_{r_{i}}-\phi_{r_{i-1}}\right)
\end{array}\right]
$$

A single observation is a function of one pose $\left(X_{r_{i}}^{T}, \phi_{r_{i}}\right)^{T}$ and one feature position $X_{f_{k}}$ which is given by

$$
H^{Z_{k}^{i}}(X)=R\left(\phi_{r_{i}}\right)^{T}\left(X_{f_{k}}-X_{r_{i}}\right)
$$

In particular, since $\phi_{r_{0}}=0$ and $X_{r_{0}}=(0,0)^{T}$, the odometry function from robot $r_{0}$ to $r_{1}$ is given by

$$
H^{O_{1}}(X)=\left[\begin{array}{c}
X_{r_{1}} \\
\phi_{r_{1}}
\end{array}\right]
$$


and the observation function from robot $r_{0}$ to $f_{k}$ is given by

$$
H^{Z_{k}^{0}}(X)=X_{f_{k}}
$$

\section{Alternative Formulation when Covariance Matrices are Spherical}

The NLLS in (3) can be simplified when matrices $P_{Z_{k_{i j}}^{i}}$ and $P_{O_{i}}$ are spherical for every $i$ and $j$.

\subsection{Definition of spherical matrices}

We first state the definitions of spherical matrices which were defined in (Wang et al. 2013).

Definition $1 A \in \mathbb{R}^{2 \times 2}$ is called spherical if it commutes with $R(\phi)$ (defined in (1)) for every $\phi$. i.e. $A R(\phi)=R(\phi) A$ for every $\phi . B \in \mathbb{R}^{3 \times 3}$ is called spherical if it has the format of $B=\operatorname{diag}(A, a)$ where $A \in \mathbb{R}^{2 \times 2}$ is spherical and $a$ is $a$ real number.

Remark 1 Every positive definite spherical matrix $A \in$ $\mathbb{R}^{2 \times 2}$ can be written as $A=a^{2} I_{2}$ for some $a \neq 0$. Furthermore, for every positive definite spherical matrix $B \in \mathbb{R}^{3 \times 3}$ we have $B=\operatorname{diag}\left(a^{2} I_{2}, b^{2}\right)$ for some non-zero $a$ and $b$.

A more general definition of spherical matrices was also introduced in (Wang et al. 2013), which will also be used in the following of this paper.

Definition 2 Let $\bar{R}_{k}(\phi)$ be the block-diagonal matrix with $k$ blocks where each block is a $2 \times 2$ rotation matrix $R(\phi)$. That is

$$
\bar{R}_{k}(\phi) \triangleq \operatorname{diag}(R(\phi), \ldots, R(\phi)) .
$$

Matrix $A \in \mathbb{R}^{2 n \times 2 m}$ is called spherical if $A \bar{R}_{m}(\phi)=$ $\bar{R}_{n}(\phi)$ A for every $\phi$.

Remark 2 The sum, difference, product, inverse, transpose of spherical matrices are also spherical matrices. This fact will be used in the proof of the main result of this paper.

When sensors used are laser range finders, the covariances can in general be approximated as spherical ones. Other sensors, in particular stereo vision, have much larger uncertainty in the depth direction, will result in covariances far from spherical. Many publicly available datasets used in SLAM have spherical covariance matrices (Kummerle, Steder, Dornhege, Ruhnke, Grisetti, Stachniss \& Kleiner 2009).

In this paper, the following strategy is used when approximating the original (non-spherical) covariance matrices $P_{Z_{k_{i j}}}, P_{O_{i}}$ as spherical matrices

$$
P_{Z_{k_{i j}}^{i}}=\operatorname{diag}\left(\bar{P}_{Z_{k_{i j}}^{i}}, \bar{P}_{Z_{k_{i j}}^{i}}\right), P_{O_{i}}=\operatorname{diag}\left(\bar{P}_{O_{i}^{x y}}, \bar{P}_{O_{i}^{x y}}, p_{O_{i}^{\phi}}\right)
$$

where $\bar{P}_{Z_{k_{i j}}^{i}}=\frac{1}{2}\left(P_{Z_{k_{i j}}^{i}}(1,1)+P_{Z_{k_{i j}}^{i}}(2,2)\right), P_{Z_{k_{i j}}^{i}}(1,1)$ and $P_{Z_{k_{i j}}^{i}}(2,2)$ are the elements in the 1-st row and 1-st column, and 2-nd row and 2-nd column of original covariance matrix $P_{Z_{k_{i j}}^{i}}$, respectively. Similarly, $\bar{P}_{O_{i}^{x y}}=\frac{1}{2}\left(P_{O_{i}}(1,1)+\right.$ $\left.P_{O_{i}}(2,2)\right), p_{O_{i}^{\phi}}=P_{O_{i}}(3,3)$.

\subsection{Alternative formulation}

When the covariance $P_{Z_{k_{i j}}^{i}}$ are $2 \times 2$ spherical positive definite matrices, we have

$$
\begin{aligned}
& \left\|Z_{k_{i j}}^{i}-H^{Z_{k_{i j}}^{i}}(X)\right\|_{P_{Z_{k_{i j}}^{i}}^{-1}}^{2} \\
= & \left\|Z_{k_{i j}}^{i}-R\left(\phi_{r_{i}}\right)^{T}\left(X_{f_{k_{i j}}}-X_{r_{i}}\right)\right\|_{P_{Z_{k_{i j}}^{i}}^{-1}}^{2} \\
= & \left\|\left(X_{f_{k_{i j}}}-X_{r_{i}}\right)-R\left(\phi_{r_{i}}\right) Z_{k_{i j}}^{i}\right\|_{P_{Z_{i j}^{i}}^{-1}}^{2} .
\end{aligned}
$$

The expression in (10) is easier to deal with than that in (9) because there is no product of variables in (10). Similarly, if we denote the odometry as $O_{i}=\left[\begin{array}{ll}O_{i}^{x y^{T}} & O_{i}^{\phi}\end{array}\right]^{T}$, then when the covariance $P_{O_{i}}$ are $3 \times 3$ spherical positive definite matrices with the format

$$
P_{O_{i}}=\operatorname{diag}\left(P_{O_{i}^{x y}}, p_{O_{i}^{\phi}}\right)
$$

with $P_{O_{i}^{x y}}$ being a $2 \times 2$ spherical positive definite matrix and $p_{O_{i}^{\phi}}>0$, then the odometry term $\left\|O_{i}-H^{O_{i}}(X)\right\|_{P_{O_{i}}^{-1}}^{2}$ in (3) becomes

$$
\begin{gathered}
\left\|O_{i}^{x y}-R\left(\phi_{r_{i-1}}\right)^{T}\left(X_{r_{i}}-X_{r_{i-1}}\right)\right\|_{P_{O_{i}^{x y}}^{-1}}^{2} \\
+p_{O_{i}^{\phi}}^{-1}\left(\operatorname{wrap}\left(O_{i}^{\phi}-\phi_{r_{i}}+\phi_{r_{i-1}}\right)\right)^{2} \\
=\left\|\left(X_{r_{i}}-X_{r_{i-1}}\right)-R\left(\phi_{r_{i-1}}\right) O_{i}^{x y}\right\|_{P_{O_{i}^{x y}}^{-1}}^{2} \\
+p_{O_{i}^{\phi}}^{-1}\left(\operatorname{wrap}\left(O_{i}^{\phi}-\phi_{r_{i}}+\phi_{r_{i-1}}\right)\right)^{2} .
\end{gathered}
$$

Thus, when $P_{Z_{k_{i j}}^{i}}$ and $P_{O_{i}}$ are all spherical, the objective function in NLLS SLAM formulation (3) can be written as

$$
\begin{aligned}
F(X)= & \sum_{i=0}^{m} \sum_{j=1}^{n_{i}}\left\|\left(X_{f_{k_{i j}}}-X_{r_{i}}\right)-R\left(\phi_{r_{i}}\right) Z_{k_{i j}}^{i}\right\|_{P_{Z_{k_{i j}}^{i}}^{-1}}^{2} \\
& +\sum_{i=1}^{m}\left\|\left(X_{r_{i}}-X_{r_{i-1}}\right)-R\left(\phi_{r_{i-1}}\right) O_{i}^{x y}\right\|_{P_{O_{i}^{x y}}^{-1}}^{2} \\
& +\sum_{i=1}^{m} p_{O_{i}^{\phi}}^{-1}\left(\operatorname{wrap}\left(O_{i}^{\phi}-\phi_{r_{i}}+\phi_{r_{i-1}}\right)\right)^{2}
\end{aligned}
$$

where $\phi_{r_{0}}=0, R\left(\phi_{r_{0}}\right)=R(0)=I$, and $X_{r_{0}}=(0,0)^{T}$. 


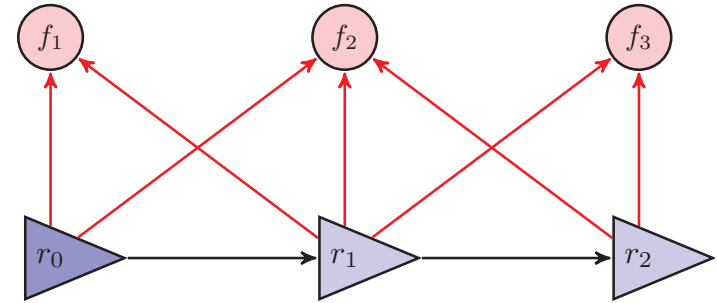

Fig. 1. An example of two-step SLAM with three features.

\section{$4 m$-Step SLAM is Equivalent to an Optimization Problem with only $m$ Variables}

To better illustrate the equivalence between optimization problem of dimensionality reduced SLAM and that of original full dimension SLAM, we first prove the equivalence for two-step SLAM case (with three robot poses $r_{0}, r_{1}, r_{2}$ ) in detail. The general $m$-step case (with $m+1$ robot poses $\left.r_{0}, r_{1}, \cdots, r_{m}\right)$ can be proved similarly.

\subsection{Two-step SLAM}

When the covariance matrices are spherical, by (13), the objective function of the two-step SLAM problem can be written in a compact form as below (note $\phi_{r_{0}}=0$ )

$$
\begin{aligned}
F\left(X_{L}, \phi_{r_{1}}, \phi_{r_{2}}\right) & =\left\|A^{T} X_{L}-\check{R}\left(\phi_{r_{0}}, \phi_{r_{1}}, \phi_{r_{2}}\right) z\right\|_{C}^{2} \\
& +p_{O_{1}^{\phi}}^{-1}\left(\operatorname{wrap}\left(O_{1}^{\phi}-\phi_{r_{1}}\right)\right)^{2} \\
& +p_{O_{2}^{\phi}}^{-1}\left(\operatorname{wrap}\left(O_{2}^{\phi}-\phi_{r_{2}}+\phi_{r_{1}}\right)\right)^{2}
\end{aligned}
$$

where $X_{L} \triangleq\left[\begin{array}{lllll}X_{f_{1}}^{T} & \ldots & X_{f_{n}}^{T} & X_{r_{1}}^{T} & X_{r_{2}}^{T}\end{array}\right]^{T}$, and

$$
A \triangleq A^{0} \otimes I_{2}
$$

where $A^{0} \in \mathbb{R}^{(n+2) \times\left(n_{0}+n_{1}+n_{2}+2\right)}$ ( $n$ is the total number of features observed, $n_{i}$ is the number of features observed from pose $\left.r_{i}, i=0,1,2\right)$ is the reduced incidence matrix of the directed graph of the SLAM problem (the direction of each edge is from the robot pose to the observed feature or from the robot pose $i$ to the robot pose $i+1$, as shown in Fig. 1). The reduced incidence matrix is obtained by deleting the row that corresponds to the "origin" in the incidence matrix of graph ${ }^{11}$. For example, for the two-step SLAM with three features as shown in Fig. 1 with pose $r_{0}$ as the "origin", the reduced incidence matrix $A^{0}$ can be written as

\footnotetext{
${ }^{1}$ For the directed graph $\mathcal{G}=(\mathcal{V}, \mathcal{E})$, the incidence matrix $\mathcal{A} \in\{0,1,-1\}^{n_{v} \times n_{e}}$ where $n_{v}=|\mathcal{V}|$ and $n_{e}=|\mathcal{E}|$. For the $k^{\prime}$ th edge $\left(i_{k}, j_{k}\right) \in \mathcal{E}, \mathcal{A}_{i_{k} k}=-1$ and $\mathcal{A}_{j_{k} k}=1$. The other elements of $\mathcal{A}$ are zero.
}

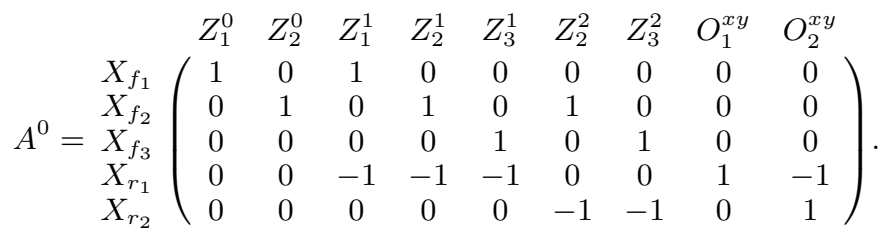

The other matrices/vectors in (14) are (note that $\phi_{r_{0}}=0$ )

$$
\begin{aligned}
& \check{R}\left(\phi_{r_{0}}, \phi_{r_{1}}, \phi_{r_{2}}\right) \\
& \triangleq \operatorname{diag}\left(\bar{R}_{n_{0}}\left(\phi_{r_{0}}\right), \bar{R}_{n_{1}}\left(\phi_{r_{1}}\right), \bar{R}_{n_{2}}\left(\phi_{r_{2}}\right), R\left(\phi_{r_{0}}\right), R\left(\phi_{r_{1}}\right)\right) \\
& =\operatorname{diag}\left(\bar{R}_{n_{0}}(0), \bar{R}_{n_{1}}\left(\phi_{r_{1}}\right), \bar{R}_{n_{2}}\left(\phi_{r_{2}}\right), R(0), R\left(\phi_{r_{1}}\right)\right) \\
& z \triangleq\left[\left(Z_{f}^{0}\right)^{T}\left(Z_{f}^{1}\right)^{T}\left(Z_{f}^{2}\right)^{T}\left(O_{1}^{x y}\right)^{T}\left(O_{2}^{x y}\right)^{T}\right]^{T} \\
& C \triangleq \operatorname{diag}\left(C_{z}^{0}, C_{z}^{1}, C_{z}^{2}, C_{p}\right)
\end{aligned}
$$

where $\bar{R}_{k}(\phi)$ is defined in (8), and

$$
\begin{aligned}
Z_{f}^{i} & =\left[\left(Z_{k_{i 1}}^{i}\right)^{T} \ldots\left(Z_{k_{i n_{i}}}^{i}\right)^{T}\right]^{T}, i=0,1,2 \\
C_{z}^{i} & =\operatorname{diag}\left(P_{Z_{k_{i 1}}^{i}}^{-1}, \ldots, P_{Z_{k_{i n_{i}}}^{i}}^{-1}\right), i=0,1,2 \\
C_{p} & =\operatorname{diag}\left(P_{O_{1}^{x y}}^{-1}, P_{O_{2}^{x y}}^{-1}\right)
\end{aligned}
$$

According to Definition 2, $A, C$ are both spherical matrices.

Remark 3 Note that when the variables $\phi_{r_{1}}, \phi_{r_{2}}$ are fixed, the function $F\left(X_{L}, \phi_{r_{1}}, \phi_{r_{2}}\right)$ in (14) becomes a quadratic function of $X_{L}$. Moreover, the matrix $A$ only depends on the structure of the graph in SLAM (i.e. which feature is observed by which pose), and $z$ is the integration of the observation and odometry data ( $x, y$ part). The special structure of the function $F\left(X_{L}, \phi_{r_{1}}, \phi_{r_{2}}\right)$ in (14) makes it possible to obtain the main result of dimensionality reduction in this paper.

The following lemma about linear least squares is well known.

Lemma 1 For the linear least-squares problem of minimizing $\left\|A^{T} X-b\right\|_{C}^{2}$ where $A, b, C$ are given, its optimal solution $X^{*}$ can be obtained by

$$
X^{*}=\left(A C^{-1} A^{T}\right)^{-1} A C^{-1} b
$$

and when substituting $X^{*}$ into $\left\|A^{T} X-b\right\|_{C}^{2}$, we can get

$$
\min _{X}\left\|A^{T} X-b\right\|_{C}^{2}=\|b\|_{Q}^{2}
$$

where

$$
Q \triangleq C-C A^{T}\left(A C A^{T}\right)^{-1} A C .
$$


Before stating the main results, we introduce more notations. Denote

$$
A_{i} \triangleq A_{i}^{0} \otimes I_{2}, \quad i=0,1,2
$$

where $A_{i}^{0}$ has the same size as $A^{0}$, and its non-zero columns are equal to the columns of $A^{0}$ in which the corresponding edges are originated from robot pose $r_{i}$. Thus

$$
A^{0}=A_{0}^{0}+A_{1}^{0}+A_{2}^{0}, \quad A=A_{0}+A_{1}+A_{2} .
$$

For example, for the two-step case as shown in Fig. 1, if we divide $A^{0}$ into five blocks as

$$
A^{0}=\left[a_{1}\left|a_{2}\right| a_{3}\left|a_{4}\right| a_{5}\right]
$$

where

$$
\begin{aligned}
& a_{1}=\left[\begin{array}{ll}
1 & 0 \\
0 & 1 \\
0 & 0 \\
0 & 0 \\
0 & 0
\end{array}\right], a_{2}=\left[\begin{array}{ccc}
1 & 0 & 0 \\
0 & 1 & 0 \\
0 & 0 & 1 \\
-1 & -1 & -1 \\
0 & 0 & 0
\end{array}\right], a_{3}=\left[\begin{array}{cc}
0 & 0 \\
1 & 0 \\
0 & 1 \\
0 & 0 \\
-1 & -1
\end{array}\right], \\
& a_{4}=\left[\begin{array}{lllll}
0 & 0 & 0 & 1 & 0
\end{array}\right]^{T}, a_{5}=\left[\begin{array}{lllll}
0 & 0 & 0 & -1 & 1
\end{array}\right]^{T} \text {, }
\end{aligned}
$$

then we have that

$$
\begin{aligned}
& A_{0}^{0}=\left[a_{1}|0| 0\left|a_{4}\right| 0\right], A_{1}^{0}=\left[\begin{array}{l|l|l|l|l}
0 & a_{2} & 0 & 0 & a_{5}
\end{array}\right], \\
& A_{2}^{0}=\left[\begin{array}{l|l|l|l|l}
0 & 0 & a_{3} & 0 & 0
\end{array}\right] \text {. }
\end{aligned}
$$

Furthermore, we define

$$
Q_{i} \triangleq C A_{i}^{T}\left(A C A^{T}\right)^{-1} A C, \quad i=0,1,2 .
$$

Now our main result about two-step SLAM is stated as follows.

Theorem 1 The two-step SLAM problem (minimizing the objective function $F\left(X_{L}, \phi_{r_{1}}, \phi_{r_{2}}\right)$ in (14)) is equivalent to a nonlinear least squares problem with only 2 variables $\phi_{r_{1}}, \phi_{r_{2}}$

$$
\min _{\phi_{r_{1}}, \phi_{r_{2}}} f_{0}\left(\phi_{r_{1}}, \phi_{r_{2}}\right)
$$

where

$$
\begin{aligned}
f_{0}\left(\phi_{r_{1}}, \phi_{r_{2}}\right) & =\left(\check{R}\left(\phi_{r_{0}}, \phi_{r_{1}}, \phi_{r_{2}}\right) z\right)^{T} Q\left(\check{R}\left(\phi_{r_{0}}, \phi_{r_{1}}, \phi_{r_{2}}\right) z\right) \\
& +\sum_{i=1}^{2} p_{O_{i}^{\phi}}^{-1}\left(\operatorname{wrap}\left(O_{i}^{\phi}-\phi_{r_{i}}+\phi_{r_{i-1}}\right)\right)^{2}
\end{aligned}
$$

with $\check{R}\left(\phi_{r_{0}}, \phi_{r_{1}}, \phi_{r_{2}}\right)$ being given in (16), and $z$ is given by (17), $Q$ is given by (23).
Furthermore, the function $f_{0}\left(\phi_{r_{1}}, \phi_{r_{2}}\right)$ can be simplified as

$$
\begin{gathered}
f_{0}\left(\phi_{r_{1}}, \phi_{r_{2}}\right)=c_{0}-2 \sum_{0 \leq i<2} \sum_{i<j \leq 2} b_{i j} \cos \left(\phi_{r_{j}}-\phi_{r_{i}}-\phi_{i j 0}\right) \\
+\sum_{i=1}^{2} p_{O_{i}^{\phi}}^{-1}\left(\operatorname{wrap}\left(O_{i}^{\phi}-\phi_{r_{i}}+\phi_{r_{i-1}}\right)\right)^{2}
\end{gathered}
$$

where $c_{0} \triangleq z^{T} C z-\sum_{i=0}^{2} b_{i i}$, and for $i, j=0,1,2$,

$$
\begin{aligned}
& b_{i j} \triangleq \sqrt{\left(z^{T} Q_{i} z_{j}\right)^{2}+\left(z^{T} Q_{i} z_{j}^{\perp}\right)^{2}} \\
& \phi_{i j 0} \triangleq \operatorname{atan} 2\left(z^{T} Q_{i} z_{j}^{\perp}, z^{T} Q_{i} z_{j}\right)
\end{aligned}
$$

with $Q_{i}$ given in (26), and $z_{j}^{\perp}$ is defined as

$$
z_{j}^{\perp} \triangleq \check{R}\left(\frac{\pi}{2}, \frac{\pi}{2}, \frac{\pi}{2}\right) z_{j}
$$

with

$$
z_{0}=\left[\begin{array}{c}
Z_{f}^{0} \\
\hline 0 \\
\hline 0 \\
\hline O_{1}^{x y} \\
0
\end{array}\right], z_{1}=\left[\begin{array}{c}
0 \\
\hline Z_{f}^{1} \\
\hline 0 \\
\hline 0 \\
\hline O_{2}^{x y}
\end{array}\right], z_{2}=\left[\begin{array}{c}
0 \\
\hline 0 \\
\hline Z_{f}^{2} \\
\hline 0 \\
\hline 0
\end{array}\right] .
$$

Proof. Apply Lemma 1, we have

$$
\begin{array}{r}
\min _{X_{L}}\left\|A^{T} X_{L}-\check{R}\left(\phi_{r_{0}}, \phi_{r_{1}}, \phi_{r_{2}}\right) z\right\|_{C}^{2} \\
=\left(\check{R}\left(\phi_{r_{0}}, \phi_{r_{1}}, \phi_{r_{2}}\right) z\right)^{T} Q \check{R}\left(\phi_{r_{0}}, \phi_{r_{1}}, \phi_{r_{2}}\right) z .
\end{array}
$$

Thus we have

$$
\begin{aligned}
\min _{X_{L}, \phi_{r_{1}}, \phi_{r_{2}}} F\left(X_{L}, \phi_{r_{1}}, \phi_{r_{2}}\right) & =\min _{\phi_{r_{1}}, \phi_{r_{2}}} \min _{L} F\left(X_{L}, \phi_{r_{1}}, \phi_{r_{2}}\right) \\
& =\min _{\phi_{r_{1}}, \phi_{r_{2}}} f_{0}\left(\phi_{r_{1}}, \phi_{r_{2}}\right)
\end{aligned}
$$

where $F\left(X_{L}, \phi_{r_{1}}, \phi_{r_{2}}\right)$ is given in (14) and $f_{0}\left(\phi_{r_{1}}, \phi_{r_{2}}\right)$ is given in (28). This completes the proof of the first part of the theorem.

Next, we prove the second part. Firstly, apart from $\bar{R}_{k}(\phi)$ defined in (8) and $\check{R}\left(\phi_{r_{0}}, \phi_{r_{1}}, \phi_{r_{2}}\right)$ defined in (16), we define

$$
\begin{aligned}
& \check{R}_{0}\left(\phi_{r_{0}}\right) \triangleq \operatorname{diag}\left(\bar{R}_{n_{0}}\left(\phi_{r_{0}}\right), 0,0, R\left(\phi_{r_{0}}\right), 0\right) \\
& \check{R}_{1}\left(\phi_{r_{1}}\right) \triangleq \operatorname{diag}\left(0, \bar{R}_{n_{1}}\left(\phi_{r_{1}}\right), 0,0, R\left(\phi_{r_{1}}\right)\right) \\
& \check{R}_{2}\left(\phi_{r_{2}}\right) \triangleq \operatorname{diag}\left(0,0, \bar{R}_{n_{2}}\left(\phi_{r_{2}}\right), 0,0\right)
\end{aligned}
$$


Note that $Q=C-C A^{T}\left(A C A^{T}\right)^{-1} A C$, and also $\check{R}^{T}\left(\phi_{r_{0}}, \phi_{r_{1}}, \phi_{r_{2}}\right) C=C \check{R}^{T}\left(\phi_{r_{0}}, \phi_{r_{1}}, \phi_{r_{2}}\right)$, we have

$z^{T} \check{R}^{T}\left(\phi_{r_{0}}, \phi_{r_{1}}, \phi_{r_{2}}\right) Q \check{R}\left(\phi_{r_{0}}, \phi_{r_{1}}, \phi_{r_{2}}\right) z=z^{T} C z$

$-z^{T}\left(\sum_{i=0}^{2} \check{R}_{i}^{T}\left(\phi_{r_{i}}\right)\right) C A^{T}\left(A C A^{T}\right)^{-1} A C \check{R}\left(\phi_{r_{0}}, \phi_{r_{1}}, \phi_{r_{2}}\right) z$.

For $i=0,1,2$,

$$
\check{R}_{i}^{T}\left(\phi_{r_{i}}\right) C=C \check{R}_{i}^{T}\left(\phi_{r_{i}}\right), \check{R}_{i}^{T}\left(\phi_{r_{i}}\right) A^{T}=A_{i}^{T} \bar{R}_{n+2}^{T}\left(\phi_{r_{i}}\right),
$$

where $A_{i}$ is defined in (24) and $\bar{R}_{k}(\phi)$ is defined in (8). Furthermore, $\left(A C A^{T}\right)^{-1} A C$ is a spherical matrix according to Remark 2, thus from Definition 2,

$\bar{R}_{n+2}^{T}\left(\phi_{r_{i}}\right)\left(A C A^{T}\right)^{-1} A C=\left(A C A^{T}\right)^{-1} A C \bar{R}_{n_{0}+n_{1}+n_{2}+2}^{T}\left(\phi_{r_{i}}\right)$.

Now we have

$$
\begin{aligned}
& \check{R}_{i}^{T}\left(\phi_{r_{i}}\right) C A^{T}\left(A C A^{T}\right)^{-1} A C \\
= & C A_{i}^{T} \bar{R}_{n+2}^{T}\left(\phi_{r_{i}}\right)\left(A C A^{T}\right)^{-1} A C \\
= & C A_{i}^{T}\left(A C A^{T}\right)^{-1} A C \bar{R}_{n_{0}+n_{1}+n_{2}+2}^{T}\left(\phi_{r_{i}}\right) \\
= & Q_{i} \bar{R}_{n_{0}+n_{1}+n_{2}+2}^{T}\left(\phi_{r_{i}}\right)
\end{aligned}
$$

where $Q_{i}$ is defined in (26). Thus,

$$
\begin{aligned}
& z^{T} \check{R}^{T}\left(\phi_{r_{0}}, \phi_{r_{1}}, \phi_{r_{2}}\right) Q \check{R}\left(\phi_{r_{0}}, \phi_{r_{1}}, \phi_{r_{2}}\right) z \\
& =z^{T} C z-z^{T}\left(\sum_{i=0}^{2} Q_{i} \bar{R}_{n_{0}+n_{1}+n_{2}+2}^{T}\left(\phi_{r_{i}}\right) \check{R}\left(\phi_{r_{0}}, \phi_{r_{1}}, \phi_{r_{2}}\right)\right) z \\
& =z^{T} C z-z^{T}\left(\sum_{i=0}^{2} Q_{i} \check{R}\left(\phi_{r_{0}}-\phi_{r_{i}}, \phi_{r_{1}}-\phi_{r_{i}}, \phi_{r_{2}}-\phi_{r_{i}}\right)\right) z .
\end{aligned}
$$

Note that $z=z_{0}+z_{1}+z_{2}$ (see (33)), we have

$$
\begin{aligned}
& z^{T}\left(\sum_{i=0}^{2} Q_{i} \check{R}\left(\phi_{r_{0}}-\phi_{r_{i}}, \phi_{r_{1}}-\phi_{r_{i}}, \phi_{r_{2}}-\phi_{r_{i}}\right)\right) z \\
= & z^{T} \sum_{i=0}^{2} Q_{i} \sum_{j=0}^{2} \check{R}_{j}\left(\phi_{r_{j}}-\phi_{r_{i}}\right) z_{j} \\
= & z^{T} \sum_{i=0}^{2} Q_{i} \sum_{j=0}^{2}\left(z_{j} \cos \left(\phi_{r_{j}}-\phi_{r_{i}}\right)+z_{j}^{\perp} \sin \left(\phi_{r_{j}}-\phi_{r_{i}}\right)\right) \\
= & \sum_{i=0}^{2} \sum_{j=0}^{2}\left(\left[\begin{array}{l}
z^{T} Q_{i} z_{j} \\
z^{T} Q_{i} z_{j}^{\perp}
\end{array}\right]^{T}\left[\begin{array}{l}
\cos \left(\phi_{r_{j}}-\phi_{r_{i}}\right) \\
\sin \left(\phi_{r_{j}}-\phi_{r_{i}}\right)
\end{array}\right]\right) .
\end{aligned}
$$

So we have

$$
\begin{aligned}
& \left\|A^{T} X_{L}-\check{R}\left(\phi_{r_{0}}, \phi_{r_{1}}, \phi_{r_{2}}\right) z\right\|_{C}^{2} \\
= & z^{T} C z-\sum_{i=0}^{2} \sum_{j=0}^{2}\left(\left[\begin{array}{l}
z^{T} Q_{i} z_{j} \\
z^{T} Q_{i} z_{j}^{\perp}
\end{array}\right]^{T}\left[\begin{array}{l}
\cos \left(\phi_{r_{j}}-\phi_{r_{i}}\right) \\
\sin \left(\phi_{r_{j}}-\phi_{r_{i}}\right)
\end{array}\right]\right) \\
= & c_{0}-\sum_{i=0}^{2} \sum_{j=0, j \neq i}^{2} b_{i j} \cos \left(\phi_{r_{j}}-\phi_{r_{i}}-\phi_{i j 0}\right)
\end{aligned}
$$

where $c_{0}=z^{T} C z-\sum_{i=0}^{2} b_{i i}, \phi_{i j 0}=0$ for $j=i$.

Notice that $z^{T} Q_{i} z_{j}=z^{T} Q_{j} z_{i}$, and

$$
\begin{aligned}
z^{T} Q_{i} z_{j}^{\perp} & =z^{T} C A_{i}^{T}\left(A C A^{T}\right)^{-1} A C \check{R}\left(\frac{\pi}{2}, \frac{\pi}{2}, \frac{\pi}{2}\right) z_{j} \\
& =-z^{T} Q_{j} z_{i}^{\perp},
\end{aligned}
$$

we have $b_{i j}=b_{j i}, \phi_{i j 0}=-\phi_{j i 0}$, and

$$
\begin{aligned}
& \left\|A^{T} X_{L}-\check{R}\left(\phi_{r_{0}}, \phi_{r_{1}}, \phi_{r_{2}}\right) z\right\|_{C}^{2} \\
= & c_{0}-2 \sum_{0 \leq i<2} \sum_{i<j \leq 2} b_{i j} \cos \left(\phi_{r_{j}}-\phi_{r_{i}}-\phi_{i j 0}\right) .
\end{aligned}
$$

Thus, $f_{0}\left(\phi_{r_{1}}, \phi_{r_{2}}\right)$ in (27) can be simplified as that in (29), this completes the proof.

\section{2 m-step SLAM}

For the $m$-step case (13), the SLAM problem can be formulated as minimizing the following objective function

$$
\begin{gathered}
F\left(X_{L}, \phi_{r_{1}}, \ldots, \phi_{r_{m}}\right)=\left\|A^{T} X_{L}-\check{R}\left(\phi_{r_{0}}, \phi_{r_{1}}, \cdots, \phi_{r_{m}}\right) z\right\|_{C}^{2} \\
+\sum_{i=1}^{m} p_{O_{i}^{\phi}}^{-1}\left(\operatorname{wrap}\left(O_{i}^{\phi}-\phi_{r_{i}}+\phi_{r_{i-1}}\right)^{2}\right)
\end{gathered}
$$

where $A=A^{0} \otimes I_{2}$, with $A^{0}$ being the reduced incidence matrix of the $m$-step SLAM graph, and $X_{L}=\left[\begin{array}{llllll}X_{f_{1}}^{T} & \ldots & X_{f_{n}}^{T} & X_{r_{1}}^{T} & \ldots & X_{r_{m}}^{T}\end{array}\right]^{T}$,

$$
\begin{gathered}
\check{R}\left(\phi_{r_{0}}, \phi_{r_{1}}, \cdots, \phi_{r_{m}}\right)=\operatorname{diag}\left(\bar{R}_{n_{0}}\left(\phi_{r_{0}}\right), \bar{R}_{n_{1}}\left(\phi_{r_{1}}\right), \ldots\right. \\
\left.\bar{R}_{n_{m}}\left(\phi_{r_{m}}\right), R\left(\phi_{r_{0}}\right), \ldots, R\left(\phi_{r_{m-1}}\right)\right) \\
z=\left[\left(Z_{f}^{0}\right)^{T} \ldots\left(Z_{f}^{m}\right)^{T}\left(O_{1}^{x y}\right)^{T} \ldots\left(O_{m}^{x y}\right)^{T}\right]^{T} \\
C=\operatorname{diag}\left(C_{z}^{0}, \ldots, C_{z}^{m}, C_{p}\right)
\end{gathered}
$$

where $Z_{f}^{i}(i=1, \cdots, m)$ is defined in (19), $C_{z}^{i}(i=$ $1, \cdots, m)$ is defined in (20), and $C_{p}=\operatorname{diag}\left(P_{O_{1}^{x y}}^{-1}, \ldots, P_{O_{m}^{x y}}^{-1}\right)$. 
Theorem 2 The m-step SLAM problem (minimizing the objective function $F\left(X_{L}, \phi_{r_{1}}, \ldots, \phi_{r_{m}}\right)$ in (39)) is equivalent to a nonlinear least squares problem with only $m$ variables $\phi_{r_{1}}, \cdots, \phi_{r_{m}}$

$$
\min _{\phi_{r_{1}}, \cdots, \phi_{r_{m}}} f_{0}\left(\phi_{r_{1}}, \cdots, \phi_{r_{m}}\right)
$$

where

$$
\begin{aligned}
& f_{0}\left(\phi_{r_{1}}, \cdots, \phi_{r_{m}}\right) \\
= & \left(\check{R}\left(\phi_{r_{0}}, \phi_{r_{1}}, \cdots, \phi_{r_{m}}\right) z\right)^{T} Q\left(\check{R}\left(\phi_{r_{0}}, \phi_{r_{1}}, \cdots, \phi_{r_{m}}\right) z\right) \\
& +\sum_{i=1}^{m} p_{O_{i}^{\phi}}^{-1}\left(\operatorname{wrap}\left(O_{i}^{\phi}-\phi_{r_{i}}+\phi_{r_{i-1}}\right)\right)^{2}
\end{aligned}
$$

with $\check{R}\left(\phi_{r_{0}}, \phi_{r_{1}}, \cdots, \phi_{r_{m}}\right)$ being given in (40), $z$ is given by (41), and $Q$ is given by (23).

Furthermore, the function $f_{0}\left(\phi_{r_{1}}, \cdots, \phi_{r_{m}}\right)$ can be simplified as

$$
\begin{aligned}
& f_{0}\left(\phi_{r_{1}}, \cdots, \phi_{r_{m}}\right)=c_{0}-2 \sum_{0 \leq i<j \leq m} b_{i j} \cos \left(\phi_{r_{j}}-\phi_{r_{i}}-\phi_{i j 0}\right) \\
& +\sum_{i=1}^{m} p_{O_{i}^{\phi}}^{-1}\left(\operatorname{wrap}\left(O_{i}^{\phi}-\phi_{r_{i}}+\phi_{r_{i-1}}\right)\right)^{2}
\end{aligned}
$$

where $c_{0}=z^{T} C z-\sum_{i=0}^{m} b_{i i}$, and for $i, j=0, \ldots, m$,

$$
\begin{aligned}
b_{i j} & \left.=\sqrt{\left(z^{T} Q_{i} z_{j}\right)^{2}+\left(z^{T} Q_{i} z_{j}^{\perp}\right.}\right)^{2} \\
\phi_{i j 0} & =\operatorname{atan} 2\left(z^{T} Q_{i} z_{j}^{\perp}, z^{T} Q_{i} z_{j}\right)
\end{aligned}
$$

with $Q_{i}=C A_{i}^{T}\left(A C A^{T}\right)^{-1} A C$, where $A_{i}, z_{j}$ are similar to those defined in Theorem 1.

Proof. The proof outline of Theorem 2 is identical to that of Theorem 1 and is omitted here.

Remark 4 From Theorem 2, it can be seen that the $m$-step point feature based SLAM problem can be formulated as minimizing a m-dimensional nonlinear function. Note that the dimension of the SLAM problem has been reduced from $3 m+2 n$ to $m$, where $n$ is the total number of features.

Remark 5 For the case of non-spherical covariance matrices, it can still be proved that the $m$-step point feature based SLAM problem is equivalent to minimizing a m-dimensional nonlinear function. However, the function will be much more complicated than that in (45).

Remark 6 In general, dimensionality reduction may not directly lead to computational saving, but for some SLAM problems, for example, when the ratio between the number of features and the number of poses is high, dimensionality reduction may improve the efficiency and convergence, this will be illustrated through examples in Section 6.
Remark 7 The idea that certain linear components can be factored out as proposed in this paper is related to that in (Zikos \& Petridis 2011). In that work the authors propose a Rao-Blackwellized particle filtering approach in which first the robot orientations are estimated using a particle filter. Then for each particle, the features and robots positions are estimated using a Kalman filter.

\section{Two-step SLAM without Orientation Part of Odom- etry}

As an application of the dimensionality reduction, we will develop an algorithm for two-step SLAM when the orientation part of the odometry is not available (or not used), that can guarantee to obtain the globally optimal solution. This is useful in building local maps for map joining algorithms. It may also lead to practical applications besides SLAM, for example, registration of three laser scans similar to Iterative Closest Point (ICP) based algorithm for registration of two laser scans.

\subsection{The optimization problem}

According to Theorem 1, the two-step SLAM problem without the orientation part of odometry is equivalent to minimizing the following objective function:

$f_{0}\left(\phi_{r_{1}}, \phi_{r_{2}}\right)=c_{0}-2 \sum_{0 \leq i<2} \sum_{i<j \leq 2} b_{i j} \cos \left(\phi_{r_{j}}-\phi_{r_{i}}-\phi_{i j 0}\right)$

Let us define

$\phi_{1} \triangleq \phi_{r_{1}}-\phi_{010}, \phi_{2} \triangleq \phi_{r_{2}}-\phi_{020}, \Delta \triangleq \phi_{020}-\phi_{010}-\phi_{120}$.

Then minimizing the above objective function is equivalent to minimizing

$$
\begin{aligned}
\bar{f}\left(\phi_{1}, \phi_{2}\right)= & -b_{01} \cos \left(\phi_{1}\right)-b_{02} \cos \left(\phi_{2}\right) \\
& -b_{12} \cos \left(\phi_{2}-\phi_{1}+\Delta\right)
\end{aligned}
$$

Since $b_{12}>0$, we can define $b_{1} \triangleq \frac{b_{01}}{b_{12}}, b_{2} \triangleq \frac{b_{02}}{b_{12}}$. Then minimizing (48) is the same as minimizing

$f\left(\phi_{1}, \phi_{2}\right)=-b_{1} \cos \left(\phi_{1}\right)-b_{2} \cos \left(\phi_{2}\right)-\cos \left(\phi_{2}-\phi_{1}+\Delta\right)$

Next, we will prove that there are at most 24 pairs of solutions to $\nabla f\left(\phi_{1}, \phi_{2}\right)=0$ in $(-\pi, \pi] \times(-\pi, \pi]$, and provide an algorithm to find all of the local minima, i.e., solutions of $\nabla f\left(\phi_{1}, \phi_{2}\right)=0$ that satisfy $\nabla^{2} f\left(\phi_{1}, \phi_{2}\right) \succ 0$. 


\subsection{Polynomial representation}

The gradient of $f\left(\phi_{1}, \phi_{2}\right)$ in (49) must vanishes at the minima:

$$
\begin{aligned}
& b_{1} \sin \left(\phi_{1}\right)-\sin \left(\phi_{2}-\phi_{1}+\Delta\right)=0 \\
& b_{2} \sin \left(\phi_{2}\right)+\sin \left(\phi_{2}-\phi_{1}+\Delta\right)=0
\end{aligned}
$$

Now let us define

$$
\phi_{3} \triangleq \phi_{2}-\phi_{1}+\Delta, \lambda \triangleq \sin \left(\phi_{3}\right)
$$

Then from (50) we have:

$$
\sin \left(\phi_{1}\right)=\frac{\lambda}{b_{1}}, \sin \left(\phi_{2}\right)=\frac{-\lambda}{b_{2}}, \sin \left(\phi_{3}\right)=\lambda
$$

By definition we have $\phi_{3}-\phi_{2}=\Delta-\phi_{1}$. By applying $\cos (\cdot)$ function on both sides, and define $c \triangleq \cos (\Delta), s \triangleq \sin (\Delta)$ we have:

$$
\cos \left(\phi_{3}\right) \cos \left(\phi_{2}\right)-c \cos \left(\phi_{1}\right)=\frac{\lambda^{2}}{b_{2}}+\frac{s}{b_{1}} \lambda
$$

Now by squaring both sides and applying (51), we get:

$$
\begin{aligned}
& \frac{\left(b_{2}^{2}-\lambda^{2}\right)\left(1-\lambda^{2}\right)}{b_{2}^{2}}+c^{2} \frac{b_{1}^{2}-\lambda^{2}}{b_{1}^{2}}-\left(\frac{\lambda^{2}}{b_{2}}+\frac{s}{b_{1}} \lambda\right)^{2} \\
& =2 c \cos \left(\phi_{1}\right) \cos \left(\phi_{2}\right) \cos \left(\phi_{3}\right)
\end{aligned}
$$

By squaring both sides again, finally, we get

$$
\begin{aligned}
& \lambda^{6}+s\left(\frac{b_{1}}{b_{2}}+\frac{b_{2}}{b_{1}}+b_{1} b_{2}\right) \lambda^{5}+\left[\frac{1}{4}\left(b_{1}^{2} b_{2}^{2}+\frac{b_{1}^{2}}{b_{2}^{2}}+\frac{b_{2}^{2}}{b_{1}^{2}}\right)\right. \\
& \left.+\left(s^{2}-\frac{1}{2}\right)\left(b_{1}^{2}+b_{2}^{2}+1\right)\right] \lambda^{4}-s\left(2-s^{2}\right) b_{1} b_{2} \lambda^{3} \\
& -\frac{1}{2} s^{2}\left(b_{1}^{2}+b_{2}^{2}+b_{1}^{2} b_{2}^{2}\right) \lambda^{2}+\frac{1}{4} s^{4} b_{1}^{2} b_{2}^{2}=0
\end{aligned}
$$

According to (51) we know that $|\lambda| \leq b_{\text {min }}$ in which $b_{\text {min }} \triangleq$ $\min \left\{1, b_{1}, b_{2}\right\}$. Therefore, we are looking for the real roots of $(53)$ in $\left[-b_{\min }, b_{\min }\right]$. Strum's theorem can be used to find the number of distinct real roots of (53) in this interval.

\subsection{Numerical algorithm}

Now we can find the globally optimal solution to the twostep SLAM problem without the orientation part of odometry using the algorithm below.

\section{Algorithm 1:}

- Step 1. Find all the real roots of the degree-6 polynomial (53) within $\left[-b_{\min }, b_{\min }\right]$ where $b_{\min } \triangleq \min \left\{1, b_{1}, b_{2}\right\}$. There are at most 6 real roots.
- Step 2. For each real root $\lambda$ within $\left[-b_{\min }, b_{\min }\right]$, compute the four possible pairs $\left(\phi_{1}, \phi_{2}\right)$ using (51).

- Step 3. For each pair $\left(\phi_{1}, \phi_{2}\right)$, check the gradient condition and the Hessian condition to see whether it is a minimum or not.

- Step 4. Compare the objective function value $f\left(\phi_{1}, \phi_{2}\right)$ for each minimum and find the global minimum.

- Step 5. For the global minimum $\left(\phi_{1}^{*}, \phi_{2}^{*}\right)$, compute

$$
\phi_{r_{1}}=\phi_{1}^{*}+\phi_{010}, \phi_{r_{2}}=\phi_{2}^{*}+\phi_{020}
$$

and then compute the rest of the optimal solution $X_{L}$ by

$$
X_{L}=\left(A C^{-1} A^{T}\right)^{-1} A C^{-1} \check{R}\left(0, \phi_{r_{1}}, \phi_{r_{2}}\right) z
$$

Remark 8 Note that the above algorithm can find all the minima and thus can be guaranteed to find the global minimum. As a byproduct, the total number of minima of the SLAM problem is also obtained.

Remark 9 The two-step SLAM problem without orientation part of odometry can be regarded as an extension of the least squares fitting of point sets (Arun, Huang \& Blostein 1987) by extending two point sets into three point sets. An optimal solution to this problem (especially in 3D) can be useful in many different applications (e.g. (Nuchter, Lingemann, Hertzberg \& Surmann 2007)).

\section{Examples}

In this section, we use simulation and experimental datasets to show the benefit of dimensionality reduction for some SLAM problems. The timing results are obtained by using simple MATLAB implementations running on an Intel Pentium CPU operating at $2.00 \mathrm{GHz}$.

\subsection{Simulation results}

\subsubsection{Improved convergency and efficiency for some sce- narios}

Assume that average $l$ features are observed by robot at each position. We show that when $l$ is large, the benefit of dimensionality reduction is clear. To show the comparison results, let the robot move 10 steps, after dimensionality reduction as proposed in this paper, the SLAM problem can be solved by minimizing objective function (45). For different $l$, Fig. 2 shows the comparison of total time required to solve the original SLAM using Gauss-Newton algorithm and that for solving the reduced dimension problem by Newton's method, from which it can be seen that when $l$ is small, e.g., less than 60 in this example, the time used by dimensionality reduction method is more than that used by original SLAM problem, however, when $l$ increases, e.g., more than 60 in this example, dimensionality reduction method is more efficient. 
Table 1

Average number of iterations (of 100 simulations) required to solve the original SLAM and the reduced dimension problem for simulations with different noise levels (in bracket: the number of simulations (out of 100) that the algoirthm does not converge).

\begin{tabular}{|c||c|c|c|c|c|}
\hline Noise level $h$ & 1 & 10 & 35 & 50 & 80 \\
\hline Original & $6(0)$ & $13(0)$ & $78(4)$ & $179(20)$ & $233(45)$ \\
\hline New & $4(0)$ & $5(0)$ & $12(0)$ & $18(0)$ & $24(0)$ \\
\hline
\end{tabular}

Noise level $h$ means adding Gaussian noises with mean 0 and standard deviation $0.1 m \times h$ to the observations of $x, y$. "Original" denotes the original SLAM using Gauss-Newton algorithm; "New" denote the new method which uses dimensionality reduction followed by Newton's method.

Furthermore, the solution to the original SLAM problem may be affected much by noise level. Let $l=44$ for example, Table 1 shows the comparison of iteration numbers for different simulations with different noise levels, it can be seen that when noise level increases (some of the noise levels are significantly larger than those in practical SLAM problems), Gauss-Newton algorithm for original SLAM problem does not converge sometimes, while the Newton's method after dimensionality reduction always converges, and the iteration number has also been reduced significantly.

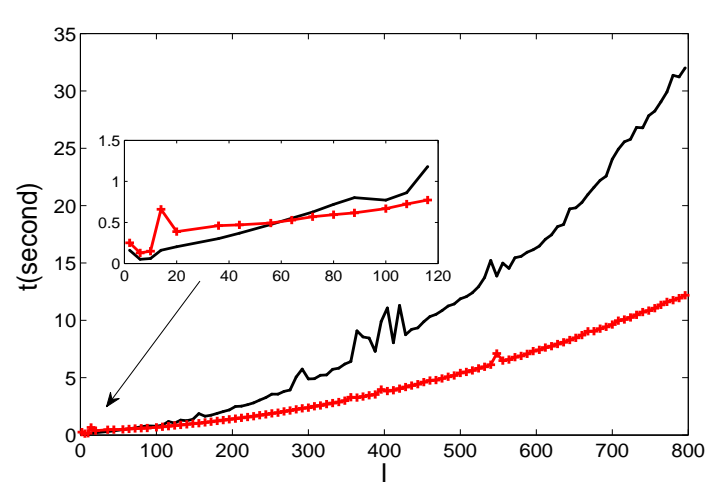

Fig. 2. Total time used by Gauss-Newton algorithm for solving the original full dimensional SLAM problem (black dots) and that using dimensionality reduction method (red crosses).

\subsubsection{Comparison of Gauss-Newton algorithm and poly- nomial method when sensor noises are large}

Now we compare the Gauss-Newton algorithm for solving the original two-step SLAM and the proposed polynomial method (Algorithm 1) for the cases when the sensor noise is large. We generate a number of datasets for the two-step SLAM without orientation part in the odometry. By changing the maximum sensor range, the number of features observed can be changed to get different simulation scenarios. Different level of noises are added to the theoretical observations and odometries, for each noise level, we generate 100 datasets and compare the results of Gauss-Newton algorithm
Table 2

The number of datasets (out of 100) when Gauss-Newton algorithm cannot converge to the global minimum within 10000 iterations (in bracket: the number of datasets that have two minima) for simulations with different noise levels.

\begin{tabular}{|c||c|c|c|c|c|}
\hline Noise level $h$ & 20 & 30 & 40 & 60 & 120 \\
\hline Sim. 1 (24 features) & $0(0)$ & $1(0)$ & $5(0)$ & $11(2)$ & $7(5)$ \\
\hline Sim. 2 (32 features) & $0(0)$ & $0(0)$ & $0(0)$ & $2(3)$ & $10(3)$ \\
\hline Sim. 3 (48 features) & $0(0)$ & $0(0)$ & $0(0)$ & $0(0)$ & $3(1)$ \\
\hline
\end{tabular}

standard deviation $0.1 m \times h$ to the observations of $x, y$.

for the original SLAM problem and the results of Algorithm 1. The comparison results are summarized in Table 2 .

From Table 2, it can be seen that when noise level is not too large, there is only one minimum and both methods give the optimal solution. However, when the noise level is very large, there is a chance that the Gauss-Newton algorithm cannot converge to the global minimum and sometimes there are two minima.

\subsection{Experimental results using the Victoria Part dataset}

\subsubsection{Result of polynomial method for two-step SLAM}

We consider the two-step SLAM problem using the first two steps of the Victoria Park dataset (Guivant \& Nebot 2001) (without using the orientation part of the odometry, the covariance matrices are set as identity matrices). There are 9 features observed for these two steps in this dataset. For this dataset, the contour of the two variable objective function $f\left(\phi_{1}, \phi_{2}\right)$ in (49) is shown in Fig. 3. The four stationary points and the unique minimum obtained by Step 3 in Algorithm 1 are shown as magenta circles and black cross, respectively. These are consistent with the contour plot of the function. The optimum result is the same as the solution obtained by using Gauss-Newton algorithm to solve the original two-step SLAM problem.

\subsubsection{Map joining result using Algorithm 1 and Linear SLAM}

For the whole Victoria Park dataset, directly applying the dimensionality reduction results does not gain computational efficiency due to the very low feature/pose ratio (299 features and 6898 poses). However, we can apply Algorithm 1 to build small local maps and apply the map joining techniques to combine the local maps to build the global map.

Recently, a new map joining algorithm, Linear SLAM, was proposed in Zhao, Huang \& Dissanayake (2013) where only solving linear least squares problems and nonlinear coordinate transformations are required to solve the map joining problem (source code available on OpenSLAM). Since Algorithm 1 guarantees to obtain the globally optimal solution 


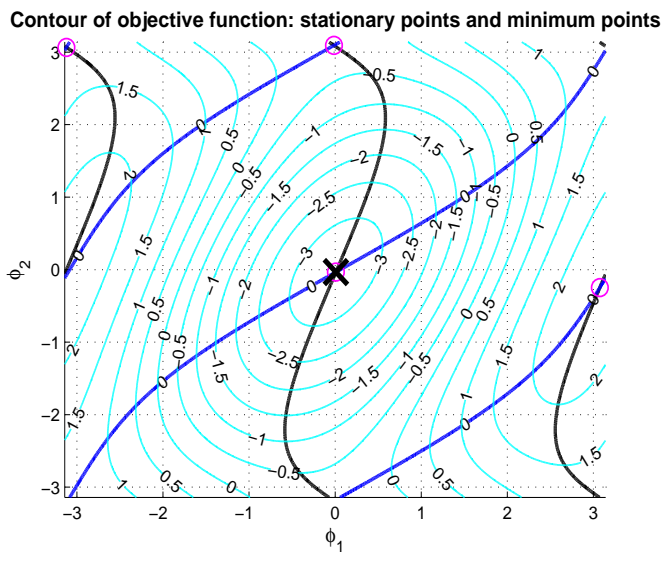

Fig. 3. Contour of the objective function $f\left(\phi_{1}, \phi_{2}\right)$ in (49).

to the local map, combining these two approaches can provide a way to obtain the global map without being trapped into a local minimum.

We first use Algorithm 1 to build 3449 local maps using the Victoria Park dataset (assuming identity covariance matrices, total time used is 112 seconds), and then use Linear SLAM algorithm to join all the local maps (time used is 25 seconds), the result is compared with that obtained using nonlinear least squares method (applied to the whole dataset with original covariance matrices) in Fig. 4. The mean absolute difference in $x$ and $y$ (for landmarks and robot poses) are $0.6307 \mathrm{~m}$ and $0.6516 \mathrm{~m}$, respectively. The mean absolute difference in robot orientation is $0.0081 \mathrm{rad}$. Note that when solving the full nonlinear least squares with original covariance matrices, the Gauss-Newton algorithm cannot converge correctly if odometry is used as the initial guess. Some other means are needed to first get a good initial value, for example, by solving the full least squares problem assuming identity covariance matrices (time used is 698 seconds, worked for this dataset). With the good initial value, the time used by Gauss-Newton algorithm is 150 seconds.

\section{Conclusions and Future Works}

This paper has proved that when the noise covariance matrices are spherical, the $m$-step SLAM problem is equivalent to an $m$-dimensional optimization problem where the objective function has a simple form. Though dimensionality reduction may not provide computational saving for all nonlinear optimization problems, we can achieve benefits such as improvement on time consumption and convergence for some SLAM problems. As an application of the dimensionality reduction result, an algorithm is developed for obtaining the globally optimal solution to the two-step SLAM problem when the orientation of the odometry is not used. Simulation and experimental dataset are used to demonstrate some benefits of the dimensionality reduction and the algorithm for two-step SLAM. Developing more efficient algorithms for solving the reduced dimensional optimization problem such as using the variable projection method (Golub

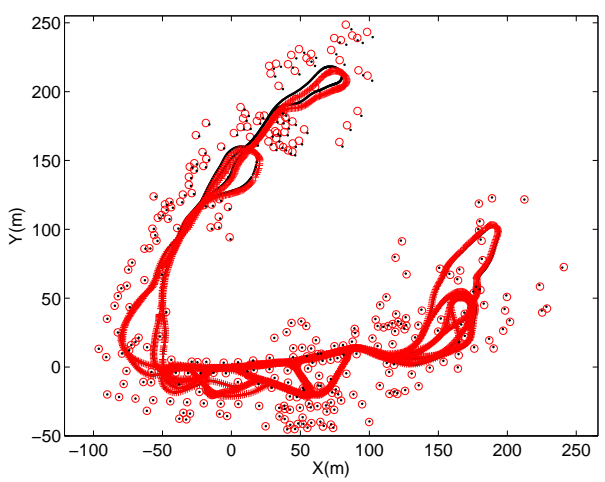

Fig. 4. Result of map joining using Algorithm 1 and Linear SLAM for Victoria Park dataset compared with full nonlinear least squares result with original covariance matrices. Red circles and black dots denote the features and robot positions obtained using least squares method, while black dots and red crosses denote features and robot positions obtained using map joining.

\& Pereyra 2003), more detailed comparisons between solving the reduced dimensional problem and solving the original SLAM problem using modern solvers, and the extension of the dimensionality reduction results to the cases of nonspherical noise covariance matrices and 3D scenarios are the next steps of research.

\section{Acknowledgements}

This work has been supported in part by the Funds of National Science of China (Grant No. 61004061, 61210306067), Australian Research Council Discovery project (DP120102786), and the German Research Foundation (DFG) under grant SFB/TR 8 Spatial Cognition.

\section{References}

Arun, K. S., Huang, T. S. \& Blostein, S. D. (1987). Least squares fitting of 3-d point sets, IEEE Transactions on Pattern Analysis and Machine Intelligence PAMI-9, 698-700.

Bailey, T. \& Durrant-Whyte, H. (2006). Simultanouse localization and mapping (slam): Part ii, IEEE Robotics \& Automation Magazine 13(2), 99-110

Carlone, L. (2013). A convergence analysis of pose graph optimization via gauss-newton methods, Proceedings of the IEEE International Conference on Robotics and Automation (ICRA), Karlsruhe, Germany, pp. 965-972.

Dellaert, F. \& Kaess, M. (2006). Square Root SAM: Simultaneous localization and mapping via square root information smoothing, Intl. J. of Robotics Research 25(12), 1181-1204.

Golub, G. \& Pereyra, V. (2003). Separable nonlinear least squares: the variable projection method and its applications, Inverse Problems 19(2), R1-R26.

Grisetti, G., Stachniss, C., Grzonka, S. \& Burgard, W. (2007). A tree parameterization for efficiently computing maximum likelihood maps using gradient descent, Proceedings of Robotics: Science and Systems, Atlanta, GA, USA. 
Guivant, J. E. \& Nebot, E. M. (2001). Optimization of the simultaneous localization and map building (slam) algorithm for real time implementation, IEEE Transactions on Robotics and Automation 17(3), 242-257.

Huang, S., Lai, Y., Frese, U. \& Dissanayake, G. (2010). How far is slam from a linear least squares problem?, IEEE/RSJ International Conference on Intelligent Robots and Systems (IROS), Taipei, Taiwan, pp. 3011-3016.

Huang, S., Wang, H., Frese, U. \& Dissanayake, G. (2012). On the number of local minima to the point feature based slam problem, IEEE International Conference on Robotics and Automation (ICRA), St. Paul, Minnesota, USA, pp. 2074-2079.

Huang, S., Wang, Z. \& Dissanayake, G. (2008). Sparse local submap joining filter for building large-scale maps, IEEE Transactions on Robotics 24(5), 1121-1130.

Kaess, M., Johannsson, H., Roberts, R., Ila, V., Leonard, J. \& Dellaert, F. (2012). isam2: Incremental smoothing and mapping using the bayes tree, International Journal of Robotics Research 31, 217-236.

Kummerle, R., Grisetti, G., Strasdat, H., Konolige, K. \& Burgard, W. (2011). G2o: A general framework for graph optimization, IEEE International Conference on Robotics and Automation (ICRA), Shanghai, China, pp. 3607-3613.

Kummerle, R., Steder, B., Dornhege, C., Ruhnke, M., Grisetti, G., Stachniss, C. \& Kleiner, A. (2009). SLAM benchmarking, http://kaspar.informatik.uni-freiburg.de/ slamEvaluation/datasets.php.

Nuchter, A., Lingemann, K., Hertzberg, J. \& Surmann, H. (2007). 6d slam-3d mapping outdoor environments, Journal of Field Robotics 24(8), 699-722.

Wang, H., Hu, G., Huang, S. \& Dissanayake, G. (2012). On the structure of nonlinearities in pose graph slam, 2012 Robotics: Science and Systems Conference (RSS), Sydney, Australia.

Wang, H., Huang, S., Frese, U. \& Dissanayake, G. (2013). The nonlinearity structure of point feature slam problems with spherical covariance matrices, Automatica 49(10), 3112-3119.

Zhao, L., Huang, S. \& Dissanayake, G. (2013). Linear slam: A linear solution to the feature-based and pose graph slam based on submap joining, 2013 IEEE/RSJ International Conference on Intelligent Robots and Systems(IROS), Tokyo, pp. 24-30.

Zikos, N. \& Petridis, V. (2011). L-slam: Reduced dimensionality fastslam with unknown data association, IEEE International Conference on Robotics and Automation (ICRA), Shanghai, China, pp. 4074-4079. 